\title{
Adaptive fault estimation for T-S fuzzy systems with unmeasurable premise variables
}

\author{
Shaokun Liu', Xiaojian Li ${ }^{1}$, Heng Wang ${ }^{1,2^{*}}$ (D) and Jingjing Yan ${ }^{1}$
}

\section{"Correspondence:}

wangheng@ise.neu.edu.cn

${ }^{1}$ College of Information Science and

Engineering, Northeastern

University, Shenyang, P.R. China

${ }^{2}$ School of Automation and

Electrical Engineering, University of Science and Technology Beijing, Beijing, P.R. China

\begin{abstract}
This paper is concerned with the fault estimation problem for a class of Takagi-Sugeno (T-S) fuzzy systems with actuator faults and sensor disturbances. Premise variables of the T-S fuzzy systems are assumed to be unmeasurable such that conventional parallel distributed compensation (PDC) methods are not applicable. A modified adaptive observer is designed to estimate states and fault parameters simultaneously. Finally, a simulation example is presented which shows the effectiveness of the proposed method.
\end{abstract}

Keywords: Takagi-Sugeno (T-S) fuzzy systems; Fault estimation; Unmeasurable premise variables

\section{Introduction}

Due to a sudden disturbance or unnecessary changes, faults are always inevitable in actual systems. Since faults deteriorate system performance and even lead to instability, fault diagnosis has been a hot research topic in the past decades. Fault diagnosis is used to check whether the system is faulty and tell when and where the faults occur; in [1], different kinds of fault detection and diagnosis methods have been reviewed, while in [2], recent developments of diagnosis and prognosis for complicated industrial systems were reviewed. Recently, a lot of research has been done in fault diagnosis for both linear and nonlinear systems in the presence of event-triggered scheme, Markovian jump phenomena, and unknown membership functions etc. For example, in [3], the problem of fault detection for nonlinear discrete-time networked systems under an event-triggered scheme was investigated, in [4], $\mathrm{H}_{2}$ fault-detection observer for two-dimensional (2-D) discrete-time Markovian jump systems was proposed, and in [5], a simultaneous fault-detection and control strategy was proposed for switched linear systems with mode-dependent average dwell-time. In [6, 7], fuzzy fault-detection observers were designed, particularly, a switching mechanism that depends on the lower and upper bounds of the unknown membership functions is provided to reduce conservatism in [6], and an integrated observer-based fault-detection scheme was proposed to meet the real-time fault-detection requirements from industrial processes in [7]. In [8], a fault-detection and -isolation (FDI) scheme for a class of Lipschitz nonlinear systems with nonlinear and unstructured modeling uncertainty was presented, and in [9], actuator stuck faults, including outage cases, were de-

(c) The Author(s) 2018. This article is distributed under the terms of the Creative Commons Attribution 4.0 International License (http://creativecommons.org/licenses/by/4.0/), which permits unrestricted use, distribution, and reproduction in any medium, provided you give appropriate credit to the original author(s) and the source, provide a link to the Creative Commons license, and indicate if changes were made. 
tected for linear state-feedback systems. Evidently, observer plays an important role in fault diagnosis, in the literature, different kinds of observers for nonlinear systems have been proposed to deal with fault-diagnosis problems, such as sliding mode observer [10], adaptive observer [11,12] and robust observer [13]. In addition, to maintain the desired performance, a robust fault estimation observer was designed in [14] based on piecewise Lyapunov functions. Fault-diagnosis schemes for T-S fuzzy model with unmeasurable premise variables were proposed based on a fuzzy PI observer and adaptive observer in [15] and [16], respectively, where faults are considered as unknown inputs in polynomials form. A multi-constrained reduced-order fault estimation observer is designed in [17] for T-S systems with actuator faults.

Note that T-S fuzzy models are capable of approximating nonlinear behaviors of smooth systems, which is achieved by blending a set of local linear models through nonlinear membership functions [18]. Some remarkable results have been reported dealing with fault-diagnosis problems of T-S fuzzy systems. For example, through considering the imperfect communication links between plant and filter, the authors in [19] designed a faultdetection filter to ensure stochastic stability of residual systems. For discrete-time T-S fuzzy system influenced by sensor faults and unknown disturbances, an $H_{-} / H_{\infty}$ robust fault-detection observer was proposed in [20] by using descriptor approach and nonquadratic Lyapunov functions, whereas the T-S fuzzy systems with unmeasurable premise variables were considered in [21]. A fault-detection filter with varying gains was designed in [6] via a switching mechanism that depends on the membership function information. In addition, adaptive fuzzy observers have been used to estimate disturbances, faults or unmodeled dynamics of practical systems, such that practical nonlinear systems can be better approximated by T-S fuzzy systems. For example, in [14], a fault estimation observer was designed for discrete-time T-S fuzzy systems via piecewise Lyapunov functions, and in [16], states and unknown inputs were estimated simultaneously by the adaptive observer designed for T-S fuzzy systems.

Note that if premise variables are allowed to be unknown or partially unknown, a wider class of nonlinear systems can be approximated by T-S fuzzy systems. However, premise variables are all assumed to be measurable in the literature when designing adaptive fuzzy observers. In this paper, a kind of loss-of-effectiveness actuator faults are considered for T-S fuzzy systems, and premise variables are assumed to be unmeasurable. In order to estimate states and fault parameters simultaneously, an adaptive observer is designed which guarantees that the estimation errors of states and fault parameters are uniformly ultimately bounded. The effectiveness of the proposed method is illustrated by a numerical example. The rest of this paper is organized as follows: In Sect. 2, some background and the system description are presented. The problem of fault estimation based on adaptive observer is addressed in Sect. 3. In Sect. 4, a numerical example is studied to illustrate the effectiveness of the proposed approach. Finally, we conclude this paper in Sect. 5.

Notation: In this paper, let $X$ be a symmetric matrix, the notation $X>0(<0)$ means that $X$ is a positive-definite(negative-definite) matrix. The superscripts " $T$ " and " -1 " stand for matrix transposition and inverse, respectively. In the symmetric matrix, an asterisk "*" is used to denote the transposed elements. For a square matrix $A, \operatorname{He}(A)$ denotes the Hermitian of the matrix $A$, that is, $\operatorname{He}(A) \triangleq A+A^{T}$. " $I$ " denotes the identity matrix with an appropriate dimension. $\|d(k)\|$ denotes the Euclidean norm of the vector $d(k) . \lambda_{\max }(A)$ and $\lambda_{\min }(A)$ represent the maximum and minimum singular value of matrix $A$, respectively. 


\section{Preliminaries and problem statement}

\subsection{System description}

Consider a T-S fuzzy system described by fuzzy IF-THEN rules. The $i$ th rule of the system is of the following form.

Plant rule $i$ : $\operatorname{IF} \xi_{1}(t)$ is $M_{1}^{i}$ and $\xi_{2}(t)$ is $M_{2}^{i}$ and $\ldots$ and $\xi_{s}(t)$ is $M_{s}^{i}$, THEN

$$
\begin{aligned}
& \dot{x}(t)=A_{i} x(t)+B_{i} u(t)+\Psi_{i}(t) \theta, \\
& y(t)=C_{i} x(t)+D_{i} d(t),
\end{aligned}
$$

where $x(t) \in \mathbb{R}^{n}$ is the state space vector, $y(t) \in \mathbb{R}^{p}$ is measurement output, and $u(t) \in \mathbb{R}^{m}$ is input vector, $d(t) \in \mathbb{R}^{l}$ is an external disturbance satisfying $d^{T}(t) d(t)<\bar{d}^{2}, \bar{d}$ is a known positive scalar. $A_{i}, B_{i}, C_{i}$ and $D_{i}$ are known parameter matrices with appropriate dimensions. $\theta \in \mathbb{R}^{m}$ is a unknown fault parameter vector. The matrix $\Psi_{i}(t) \in \mathbb{R}^{n} \times \mathbb{R}^{m}$ is the known signal. $\xi(t)=\left[\xi_{1}(t), \xi_{2}(t), \ldots, \xi_{s}(t)\right]$ are the premise variables which can be measurable such as $\{u(t), y(t)\}$ or unmeasurable as the state $\mathrm{x}(\mathrm{t})$ of the system, $M_{j}^{i}(i=1,2, \ldots, q$, $j=1,2, \ldots, s)$ are the fuzzy sets, $q$ is the number of IF-THEN rules, and $s$ is the number of premise variables. Throughout this paper, it is assumed that the premise variable of the fuzzy system is unmeasurable.

By using "fuzzy blending" of each individual plant rule (local model), the global dynamics of T-S fuzzy system (1) can be described as follows:

$$
\begin{aligned}
& \dot{x}(t)=\sum_{i=1}^{q} \mu_{i}(x(t))\left(A_{i} x(t)+B_{i} u(t)+\Psi_{i}(t) \theta\right), \\
& y(t)=\sum_{i=1}^{q} \mu_{i}(x(t))\left(C_{i} x(t)+D_{i} d(t)\right),
\end{aligned}
$$

where $\mu_{i}(x(t))=h_{i}(x(t)) / \sum_{i=1}^{q} h_{i}(x(t)), h_{i}(x(t))=\prod_{j=1}^{s} M_{j}^{i}\left(x_{j}(t)\right)$, and $M_{j}^{i}\left(x_{j}(t)\right)$ is the grade of the membership function of $x_{j}(t)$ in $M_{j}^{i}$. It is assumed that $h_{i}(x(t)) \geq 0(i=1,2, \ldots, q)$. Hence, we see that the normalized membership function $\mu_{i}(x(t))$ satisfies $\mu_{i}(x(t)) \geq 0$ and $\sum_{i=1}^{q} \mu_{i}(x(t))=1$ for all $t$.

In this paper, we use $\Psi_{i}(t) \theta$ to represent possible actuator faults, i.e., the loss-ofeffectiveness actuator fault as considered in $[22,23]$. The actuator fault model is as follows:

$$
u_{d}^{F}(t)=\rho_{d} u_{d}(t), \quad d=1,2, \ldots, m,
$$

where $\rho_{d} \in[0,1]$ is the unknown actuator efficiency factor. Note that the system is faultfree when $\rho_{d}=1$. The actuator fault model can be denoted as

$$
u^{F}(t)=\rho u(t)
$$

where $\rho=\operatorname{diag}\left\{\rho_{1}, \rho_{2}, \ldots, \rho_{m}\right\}$. The control input term $B_{i} u^{F}(t)$ becomes $B_{i} \rho u(t)$. For the sake of analysis, define $B_{i}=\left[\begin{array}{llll}B_{i 1} & B_{i 2} & \ldots & B_{i m}\end{array}\right], \bar{u}(t)=\operatorname{diag}\left\{u_{1}(t), u_{2}(t), \ldots, u_{m}(t)\right\}, \Psi_{i}(t)=$ $\left[\begin{array}{llll}\Psi_{i 1}(t) & \Psi_{i 2}(t) \ldots \Psi_{i n}(t)\end{array}\right]^{T}, \theta=\left[\begin{array}{llll}\theta_{1} & \theta_{2} & \ldots & \theta_{m}\end{array}\right]^{T}$. Let $\Psi_{i}(t)=B_{i} \bar{u}(t), \theta_{k}=\rho_{k}-1, k=1,2, \ldots, m$ such that the input term $\Psi_{i}(t) \theta$ denotes the loss of effectiveness model of the actuator. 


\subsection{Adaptive observer}

In this subsection, an adaptive observer is presented to jointly estimate states and fault parameters of T-S system (2), based on which the estimated states are chosen as premise variables.

Motivated by [24], a modified adaptive observer is designed to perform fault estimation. From the previous description, the rules of the distributed observer is shown as follows.

Observer rule $i$ : $\operatorname{IF} \hat{x}_{1}(t)$ is $M_{1}^{i}$ and $\hat{x}_{2}(t)$ is $M_{2}^{i}$ and $\ldots$ and $\hat{x}_{s}(t)$ is $M_{s}^{i}$, THEN

$$
\begin{aligned}
& \dot{\Upsilon}(t)=\left(A_{i}-K C_{i}\right) \Upsilon(t)+\Psi_{i}(t), \\
& \dot{\hat{x}}(t)=A_{i} \hat{x}(t)+B_{i} u(t)+\Psi_{i}(t) \hat{\theta}(t)+\left(K+\Upsilon(t) \Gamma \Upsilon(t)^{T} C_{i}^{T}\right)\left(y(t)-C_{i} \hat{x}(t)\right), \\
& \dot{\hat{\theta}}(t)=\Gamma \Upsilon(t)^{T} C_{i}^{T}\left(y(t)-C_{i} \hat{x}(t)\right),
\end{aligned}
$$

where $\hat{x}(t)$ is the observer state, $\hat{\theta}(t) \in \mathbb{R}^{n}$ is an estimate of $\theta$. $\Upsilon(t)$ is generated by linearly filtering $\Psi_{i}(t)$. $\Gamma \in \mathbb{R}^{m} \times \mathbb{R}^{m}$ is chosen to be a symmetric and positive-definite matrix, tuned to balance the convergence speeds of the states and fault parameters. $K$ is the gain matrix to be determined such that $A_{i}-K C_{i}$ is stable.

Similar to (2), the fuzzy adaptive observer is developed by

$$
\begin{aligned}
\dot{\Upsilon}(t)= & \sum_{i=1}^{r} \mu_{i}(\hat{x}(t))\left(\left(A_{i}-K C_{i}\right) \Upsilon(t)+\Psi_{i}(t)\right), \\
\dot{\hat{x}}(t)= & \sum_{i=1}^{r} \mu_{i}(\hat{x}(t))\left(A_{i} \hat{x}(t)+B_{i} u(t)+\Psi_{i}(t) \hat{\theta}(t)\right. \\
& \left.+\left(K+\Upsilon(t) \Gamma \Upsilon(t)^{T} C_{i}^{T}\right)\left(y(t)-C_{i} \hat{x}(t)\right)\right), \\
\dot{\hat{\theta}}(t)= & \sum_{i=1}^{r} \mu_{i}(\hat{x}(t))\left(\Gamma \Upsilon(t)^{T} C_{i}^{T}\left(y(t)-C_{i} \hat{x}(t)\right)\right) .
\end{aligned}
$$

In order to guarantee the convergence of fault estimate error, the following assumption is required in this paper.

Assumption 1 Assume that $\Psi_{i}(t)$ is persistently exciting, for some positive constants $\alpha$, $T$ and for all $t \geq t_{0}$, the following inequality holds:

$$
\int_{t}^{t+T} \Upsilon(\tau)^{T} C_{i}^{T} C_{i} \Upsilon(\tau) \geq \alpha I
$$

Assumption 2 Control input $u(t)$ has been designed to ensure the stability of the T-S fuzzy system in both fault-free and faulty case.

Assumption 3 The normalized membership functions $\mu_{i}(x(t))$ with the unmeasurable premise variable $x(t)$ are the Lipschitz continuous functions with Lipschitz constants $\varrho_{i}$ on a compact region $\mathbb{D}$, such that

$$
\left|\mu_{i}(x(t))-\mu_{i}(\hat{x}(t))\right| \leq \varrho_{i}\|x(t)-\hat{x}(t)\|, \quad i=1,2, \ldots, q .
$$


Let $\Delta(x(t), \hat{x}(t), \theta)=\left(\sum_{i=1}^{r} \mu_{i}(x(t))-\sum_{i=1}^{r} \mu_{i}(\hat{x}(t))\right)\left(A_{i} x(t)+B_{i} u(t)+\Psi_{i}(t) \theta\right)$, applying Assumption 3 , we have that there exists a positive scalar $\beta$ such that

$$
\Delta(x(t), \hat{x}(t), \theta)^{T} \Delta(x(t), \hat{x}(t), \theta) \leq \beta^{2} \tilde{x}(t)^{T} \tilde{x}(t)
$$

which has been used in $[13,15]$. Then we have the following assumption.

Assumption 4 There exist positive scalars $\varepsilon_{i}, \pi$ such that

$$
\begin{aligned}
& C_{i}^{T} C_{i}>\frac{\beta^{2}}{\varepsilon_{i}} I, \\
& D_{i} D_{i}^{T}<\frac{1}{\pi} I,
\end{aligned}
$$

where $\beta$ satisfies (11).

Remark 1 Assumption 1 is the persistent excitation condition, which has been presented in [24-26]. Assumption 2 and Assumption 3 are widely used in the fault-diagnosis literature $[13,15,16]$. In Assumption $4, C_{i}$ needs to be full column rank. Since $\beta$ is a positive bounded scalar, it is easy to find a positive scalar $\varepsilon$ and $\pi$ such that conditions (12) and (13) hold.

The following lemma is essential for later development.

Lemma 1 ([27]) For any matrices $X, Y$ with appropriate dimensions, the following inequality holds for any positive scalars $\varepsilon$ :

$$
X^{T} Y+Y^{T} X \leq \varepsilon X^{T} X+\frac{1}{\varepsilon} Y^{T} Y
$$

Now, the main problem studied in this paper is summarized as follows.

Problem For T-S fuzzy system (2) with actuator faults and external disturbances (including loss of effectiveness and bias), the main objective of this paper is to design an adaptive observer such that states and fault parameters can be estimated simultaneously.

\section{Fault estimation based on an adaptive observer}

In this section, we will analyze the behavior of the proposed adaptive observer (6) for fuzzy system (2) with the help of Lyapunov-Krasovskii functional approach.

Theorem 1 Consider T-S fuzzy system (2) and adaptive observer (6)-(8), the state estimation errors $\tilde{x}(t)$ and fault estimated errors $\tilde{\theta}(t)$ are uniformly ultimately bounded if there exist symmetric positive matrix variables $P, Q$ and positive scalars $\varsigma_{i}, \varepsilon_{i}$ such that the following LMI (linear matrix inequality) holds for $i=1,2, \ldots, q$ :

$$
\left[\begin{array}{ccc}
\mathrm{He}\left(P A_{i}-Q C_{i}\right)+C_{i}^{T} C_{i} & Q D_{i} & P \\
* & -\frac{1}{\varsigma_{i}} I & 0 \\
* & * & -\frac{1}{\varepsilon_{i}} I
\end{array}\right]<0
$$

where $K=P^{-1} Q$. 
Proof Combining (7) and (8), we have

$$
\dot{\hat{x}}(t)=\sum_{i=1}^{r} \mu_{i}(\hat{x}(t))\left(A_{i} \hat{x}(t)+B_{i} u(t)+\Psi_{i}(t) \hat{\theta}(t)+\Upsilon(t) \dot{\hat{\theta}}(t)\right)+K\left(y(t)-C_{i} \hat{x}(t)\right) .
$$

Let

$$
\tilde{x}(t)=x(t)-\hat{x}(t), \quad \tilde{\theta}(t)=\theta-\hat{\theta}(t) .
$$

Recall system model (2) and notice that $\dot{\theta}=0$, then

$$
\begin{aligned}
& \dot{\tilde{x}}(t)=\dot{x}(t)-\dot{\hat{x}}(t) \\
& =\sum_{i=1}^{r} \mu_{i}(x(t))\left(A_{i} x(t)+B_{i} u(t)+\Psi_{i}(t) \theta\right) \\
& -\sum_{i=1}^{r} \mu_{i}(\hat{x}(t))\left(A_{i} \hat{x}(t)+B_{i} u(t)+\Psi_{i}(t) \hat{\theta}(t)\right. \\
& \left.+\left(K+\Upsilon(t) \Gamma \Upsilon(t)^{T} C_{i}^{T}\right)\left(y(t)-C_{i} \hat{x}(t)\right)\right) \\
& =\sum_{i=1}^{r} \mu_{i}(x(t))\left(A_{i} x(t)+B_{i} u(t)+\Psi_{i}(t) \theta\right) \\
& -\sum_{i=1}^{r} \mu_{i}(\hat{x}(t))\left(A_{i} x(t)+B_{i} u(t)+\Psi_{i}(t) \theta\right) \\
& +\sum_{i=1}^{r} \mu_{i}(\hat{x}(t))\left(A_{i} x(t)+B_{i} u(t)+\Psi_{i}(t) \theta\right) \\
& -\sum_{i=1}^{r} \mu_{i}(\hat{x}(t))\left(A_{i} \hat{x}(t)+B_{i} u(t)+\Psi_{i}(t) \hat{\theta}(t)\right. \\
& \left.+\left(K+\Upsilon(t) \Gamma \Upsilon(t)^{T} C_{i}^{T}\right)\left(y(t)-C_{i} \hat{x}(t)\right)\right) \\
& =\sum_{i=1}^{r} \mu_{i}(\hat{x}(t))\left(\left(A_{i}-K C_{i}\right) \tilde{x}(t)+\Psi_{i}(t) \tilde{\theta}(t)+\Upsilon(t) \dot{\tilde{\theta}}(t)\right. \\
& \left.-K D_{i} d(t)+\Delta(x(t), \hat{x}(t), \theta)\right),
\end{aligned}
$$

where $\Delta(x(t), \hat{x}(t), \theta)=\left(\sum_{i=1}^{r} \mu_{i}(x(t))-\sum_{i=1}^{r} \mu_{i}(\hat{x}(t))\right)\left(A_{i} x(t)+B_{i} u(t)+\Psi_{i}(t) \theta\right)$, and $K$ is defined in (5).

Define

$$
\eta(t)=\tilde{x}(t)-\Upsilon(t) \tilde{\theta}(t)
$$

and take the first-order derivative of $\eta(t)$; we have

$$
\begin{aligned}
\dot{\eta}(t)= & \sum_{i=1}^{r} \mu_{i}(\hat{x}(t))\left(\left(A_{i}-K C_{i}\right) \tilde{x}(t)+\Psi_{i}(t) \tilde{\theta}(t)+\Upsilon(t) \dot{\tilde{\theta}}(t)-K D_{i} d(t)\right. \\
& +\Delta(x(t), \hat{x}(t), \theta)-\dot{\Upsilon}(t) \tilde{\theta}(t)-\Upsilon(t) \dot{\tilde{\theta}}(t))
\end{aligned}
$$




$$
\begin{aligned}
= & \sum_{i=1}^{r} \mu_{i}(\hat{x}(t))\left(\left(A_{i}-K C_{i}\right) \tilde{x}(t)+\Psi_{i}(t) \tilde{\theta}(t)-K D_{i} d(t)\right. \\
& +\Delta(x(t), \hat{x}(t), \theta)-\dot{\Upsilon}(t) \tilde{\theta}(t)) \\
= & \sum_{i=1}^{r} \mu_{i}(\hat{x}(t))\left(\left(A_{i}-K C_{i}\right) \eta(t)+\left(\left(A_{i}-K C_{i}\right) \Upsilon(t)+\Psi_{i}(t)-\dot{\Upsilon}(t)\right) \tilde{\theta}(t)\right. \\
& \left.-K D_{i} d(t)+\Delta(x(t), \hat{x}(t), \theta)\right) .
\end{aligned}
$$

Substituting (6) into (24) yields

$$
\dot{\eta}(t)=\sum_{i=1}^{r} \mu_{i}(\hat{x}(t))\left(\left(A_{i}-K C_{i}\right) \eta(t)-K D_{i} d(t)+\Delta(x(t), \hat{x}(t), \theta)\right) .
$$

Since $\dot{\theta}=0$, replacing $\tilde{x}(t)$ by $\eta(t)$ and $\tilde{\theta}(t)$ according to (22), we have

$$
\begin{aligned}
\dot{\tilde{\theta}}(t)= & \sum_{i=1}^{r} \mu_{i}(\hat{x}(t))\left(-\Gamma \Upsilon(t)^{T} C_{i}^{T} C_{i} \eta(t)-\Gamma \Upsilon(t)^{T} C_{i}^{T} C_{i} \Upsilon(t) \tilde{\theta}(t)\right. \\
& \left.-\Gamma \Upsilon(t)^{T} C_{i}^{T} D_{i} d(t)\right) .
\end{aligned}
$$

Choose the following Lyapunov function candidate:

$$
V(t)=\eta^{T}(t) P \eta(t)+\tilde{\theta}^{T}(t) \Gamma^{-1} \tilde{\theta}(t)
$$

where $P$ is a symmetric positive-defined matrix, and $\Gamma$ is the matrix gain used in (5). Then

$$
\begin{aligned}
\dot{V}(t)= & 2 \eta(t)^{T} P \dot{\eta}(t)+2 \tilde{\theta}(t)^{T} \Gamma^{-1} \dot{\tilde{\theta}}(t) \\
= & \sum_{i=1}^{r} \mu_{i}(\hat{x}(t))\left(2 \eta(t)^{T} P\left(\left(A_{i}-K C_{i}\right) \eta(t)-K D_{i} d(t)+\Delta(x(t), \hat{x}(t), \theta)\right)\right. \\
& -2 \tilde{\theta}(t)^{T} \Gamma^{-1}\left(\Gamma \Upsilon(t)^{T} C_{i}^{T} C_{i} \eta(t)+\Gamma \Upsilon(t)^{T} C_{i}^{T} C_{i} \Upsilon(t) \tilde{\theta}(t)\right. \\
& \left.+\Gamma \Upsilon(t)^{T} C_{i}^{T} D_{i} d(t)\right) \\
= & \sum_{i=1}^{r} \mu_{i}(\hat{x}(t))\left(\eta(t)^{T} \operatorname{He}\left(P\left(A_{i}-K C_{i}\right)\right) \eta(t)-2 \eta(t)^{T} P K D_{i} d(t)\right. \\
& +2 \eta(t)^{T} P \Delta(x(t), \hat{x}(t), \theta)-2 \tilde{\theta}(t)^{T} \Upsilon(t)^{T} C_{i}^{T} C_{i} \eta(t) \\
& \left.-2 \tilde{\theta}(t)^{T} \Upsilon(t)^{T} C_{i}^{T} C_{i} \Upsilon(t) \tilde{\theta}(t)-2 \tilde{\theta}(t)^{T} \Upsilon(t)^{T} C_{i}^{T} D_{i} d(t)\right) .
\end{aligned}
$$

From Lemma 1, it can be seen that

$$
\begin{aligned}
& -2 \eta(t)^{T} P K D_{i} d(t) \leq \varsigma \eta(t)^{T} P K D_{i} D_{i}^{T} K^{T} P \eta(t)+\frac{1}{\zeta} d^{T}(t) d(t), \\
& 2 \eta(t)^{T} P \Delta(x(t), \hat{x}(t), \theta) \leq \varepsilon \eta(t)^{T} P P \eta(t)+\frac{1}{\varepsilon} \Delta(x(t), \hat{x}(t), \theta)^{T} \Delta(x(t), \hat{x}(t), \theta), \\
& -2 \tilde{\theta}(t)^{T} \Upsilon(t)^{T} C_{i}^{T} D_{i} d(t) \leq \pi \tilde{\theta}(t)^{T} \Upsilon(t)^{T} C_{i}^{T} D_{i} D_{i}^{T} C_{i} \Upsilon(t) \tilde{\theta}(t)+\frac{1}{\pi} d^{T}(t) d(t),
\end{aligned}
$$


From Assumption 2, we see that both $x(t)$ and $u(t)$ are bounded, then from Assumption 3, we have

$$
\begin{aligned}
& d^{T}(t) d(t) \leq \bar{d}^{2}, \\
& \Delta(x(t), \hat{x}(t), \theta)^{T} \Delta(x(t), \hat{x}(t), \theta) \leq \beta^{2} \tilde{x}(t)^{T} \tilde{x}(t) .
\end{aligned}
$$

Substituting (29)-(31) into the derivative of Lyapunov function (28), we have

$$
\begin{aligned}
\dot{V}(t) \leq & \sum_{i=1}^{r} \mu_{i}(\hat{x}(t))\left(\eta(t)^{T}\left(\operatorname{He}\left(P\left(A_{i}-K C_{i}\right)\right)+\varepsilon P P+\varsigma P K D_{i} D_{i}^{T} K^{T} P\right) \eta(t)\right. \\
& +\frac{\beta^{2}}{\varepsilon} \tilde{x}(t)^{T} \tilde{x}(t)-2 \tilde{\theta}(t)^{T} \Upsilon(t)^{T} C_{i}^{T} C_{i} \eta(t)-2 \tilde{\theta}(t)^{T} \Upsilon(t)^{T} C_{i}^{T} C_{i} \Upsilon(t) \tilde{\theta}(t) \\
& \left.+\frac{\bar{d}^{2}}{\varsigma}+\pi \tilde{\theta}(t)^{T} \Upsilon(t)^{T} C_{i}^{T} D_{i} D_{i}^{T} C_{i} \Upsilon(t) \tilde{\theta}(t)+\frac{1}{\pi} d^{T}(t) d(t)\right) .
\end{aligned}
$$

Since

$$
\tilde{x}(t)^{T} \tilde{x}(t)=\eta(t)^{T} \Upsilon(t) \tilde{\theta}(t)+\tilde{\theta}(t)^{T} \Upsilon(t)^{T} \eta(t)+\tilde{\theta}(t)^{T} \Upsilon(t)^{T} \Upsilon(t) \tilde{\theta}(t)+\eta^{T}(t) \eta(t)
$$

we have

$$
\begin{aligned}
\dot{V}(t) \leq & \sum_{i=1}^{r} \mu_{i}(\hat{x}(t))\left(\eta(t)^{T}\left(\operatorname{He}\left(P\left(A_{i}-K C_{i}\right)\right)+\varepsilon P P+\varsigma P K D_{i} D_{i}^{T} K^{T} P+\frac{\beta^{2}}{\varepsilon} I\right) \eta(t)\right. \\
& +\frac{\beta^{2}}{\varepsilon} \eta(t)^{T} \Upsilon(t) \tilde{\theta}(t)+\frac{\beta^{2}}{\varepsilon} \tilde{\theta}(t)^{T} \Upsilon(t)^{T} \eta(t)+\frac{\beta^{2}}{\varepsilon} \tilde{\theta}(t)^{T} \Upsilon(t)^{T} \Upsilon(t) \tilde{\theta}(t) \\
& -2 \tilde{\theta}(t)^{T} \Upsilon(t)^{T} C_{i}^{T} C_{i} \eta(t)-2 \tilde{\theta}(t)^{T} \Upsilon(t)^{T} C_{i}^{T} C_{i} \Upsilon(t) \tilde{\theta}(t)+\frac{1}{\varsigma} d^{T}(t) d(t) \\
& \left.+\pi \tilde{\theta}(t)^{T} \Upsilon(t)^{T} C_{i}^{T} D_{i} D_{i}^{T} C_{i} \Upsilon(t) \tilde{\theta}(t)+\frac{1}{\pi} d^{T}(t) d(t)\right) \\
\leq & \sum_{i=1}^{r} \mu_{i}(\hat{x}(t))\left(\left[\begin{array}{l}
\eta(t) \\
\tilde{\theta}(t)
\end{array}\right]^{T} \Omega_{i}\left[\begin{array}{c}
\eta(t) \\
\tilde{\theta}(t)
\end{array}\right]\right)+\left(\frac{1}{\varsigma}+\frac{1}{\pi}\right) \bar{d}^{2}
\end{aligned}
$$

where

$$
\begin{aligned}
& \Omega_{i}=\left[\begin{array}{cc}
\Sigma_{11}+\frac{\beta^{2}}{\varepsilon} I & \frac{\beta^{2}}{\varepsilon} \Upsilon(t)-C_{i}^{T} C_{i} \Upsilon(t) \\
* & \frac{\beta^{2}}{\varepsilon} \Upsilon(t)^{T} \Upsilon(t)-2 \Upsilon(t)^{T} C_{i}^{T} C_{i} \Upsilon(t)+\pi \Upsilon(t)^{T} C_{i}^{T} D_{i} D_{i}^{T} C_{i} \Upsilon(t)
\end{array}\right], \\
& \Sigma_{11}=\operatorname{He}\left(P\left(A_{i}-K C_{i}\right)\right)+\varepsilon P P+\varsigma P K D_{i} D_{i}^{T} K^{T} P .
\end{aligned}
$$

Note that $\Omega_{i}=\Omega_{i 1}+\Omega_{i 2}$, where

$$
\begin{aligned}
& \Omega_{i 1}=\left[\begin{array}{cc}
\Sigma_{11}+C_{i}^{T} C_{i} & 0 \\
0 & -\Upsilon(t)^{T} C_{i}^{T}\left(I-\pi D_{i} D_{i}^{T}\right) C_{i} \Upsilon(t)
\end{array}\right], \\
& \Omega_{i 2}=\left[\begin{array}{cc}
\frac{\beta^{2}}{\varepsilon} I-C_{i}^{T} C_{i} & \left(\frac{\beta^{2}}{\varepsilon} I-C_{i}^{T} C_{i}\right) \Upsilon(t) \\
* & \Upsilon(t)^{T}\left(\frac{\beta^{2}}{\varepsilon} I-C_{i}^{T} C_{i}\right) \Upsilon(t)
\end{array}\right]=\left[\begin{array}{c}
I \\
\Upsilon^{T}(t)
\end{array}\right]\left(\frac{\beta^{2}}{\varepsilon} I-C_{i}^{T} C_{i}\right)\left[\begin{array}{ll}
I & \Upsilon(t)]
\end{array}\right.
\end{aligned}
$$


From Assumption 4, we have $\frac{\beta^{2}}{\varepsilon} I-C_{i}^{T} C_{i}<0$, thus, $\Omega_{i 2} \leq 0$. From Assumption 1 and 4 , we have $-\Upsilon(t)^{T} C_{i}^{T}\left(I-\pi D_{i} D_{i}^{T}\right) C_{i} \Upsilon(t)<0$, then $\Omega_{i 1}<0$ if and only if $\Sigma_{11}+C_{i}^{T} C_{i}<0$ holds which is equivalent to the following inequality by using the Schur complement lemma:

$$
\left[\begin{array}{ccc}
\mathrm{He}\left(P\left(A_{i}-K C_{i}\right)\right)+C_{i}^{T} C_{i} & P K D_{i} & P \\
* & -\frac{1}{5} I & 0 \\
* & * & -\frac{1}{\varepsilon} I
\end{array}\right]<0 .
$$

Summarily, we see that if inequality (37) holds, we have $\Omega_{i 1}<0$, since $\Omega_{i 2} \leq 0$ holds, we have $\Omega_{i}<0$. Recall (36), we have

$$
\dot{V}(t) \leq-\lambda_{\min }\left(-\Omega_{i}\right)\left(\|\eta(t)\|^{2}+\|\tilde{\theta}(t)\|^{2}\right)+\left(\frac{1}{\varsigma}+\frac{1}{\pi}\right) \bar{d}^{2} .
$$

Let $\vartheta(t)=\left[\eta^{T}(t) \tilde{\theta}^{T}(t)\right]^{T}$, we have

$$
\dot{V}(t)<0, \quad \forall\|\vartheta(t)\|^{2}>\frac{(\varsigma+\pi) \bar{d}^{2}}{\varsigma \pi \lambda_{\min }\left(-\Omega_{i}\right)} .
$$

Thus, if inequality (37) holds, systems (25) and (26) are uniformly ultimately bounded. Let $Q=P K$, (37) becomes (15), this completes the proof.

Remark 2 Note that if condition (15) holds, the gain matrix $K$ can be obtained by solving LMI (15) through using MATLAB LMI toolbox, then the state $x(t)$ and fault parameter $\theta(t)$ can be estimated by the adaptive observer (6)-(8).

\section{Numerical example}

In this section, a numerical example is used to illustrate the effectiveness of proposed fault estimation method. Consider system model (1) with the following system of matrices:

$$
\begin{array}{ll}
A_{1}=\left[\begin{array}{cc}
0 & 2 \\
-1 & -10
\end{array}\right], \quad A_{2}=\left[\begin{array}{cc}
0 & 2 \\
-5 & -10
\end{array}\right], \quad B_{1}=B_{2}=\left[\begin{array}{ll}
1 & 0 \\
1 & 2
\end{array}\right], \\
C_{1}=C_{2}=\left[\begin{array}{cc}
0 & 10 \\
8 & 0
\end{array}\right], \quad D_{1}=D_{2}=\left[\begin{array}{cc}
10 & 5 \\
15 & 10
\end{array}\right] .
\end{array}
$$

The membership functions are assumed to depend on the first component $x_{1}$ of the unmeasured state vector $x$, which are

$$
\mu_{1}(x)=1-\frac{x_{1}^{2}}{25}, \quad \mu_{2}(x)=1-\mu_{1}(x)
$$

The initial states are

$$
\begin{aligned}
& x(0)=\left[\begin{array}{ll}
0.2 & 0
\end{array}\right]^{T}, \quad \hat{x}(0)=\left[\begin{array}{ll}
0.2 & 0
\end{array}\right]^{T}, \quad \hat{\theta}(0)=\left[\begin{array}{ll}
0.3 & 0.3
\end{array}\right]^{T}, \\
& \Upsilon_{1}(0)=\Upsilon_{2}(0)=\left[\begin{array}{ll}
0 & 0 \\
0 & 0
\end{array}\right]^{T} .
\end{aligned}
$$


The adaptive observer parameter $\Gamma$ used in this simulation is

$$
\Gamma=\left[\begin{array}{cc}
0.8 & 0 \\
0 & 0.5
\end{array}\right]
$$

In order to ensure that the system is stable for both faulty and fault-free cases, the controller is designed as $u(t)=\sum_{i=1}^{r} \mu_{i}(\hat{x})\left(L_{i} y(t)+r(t)\right)$, where $L_{i}$ is the feedback gain, $r(t)$ is the reference signal. In this example, the controller parameters are chosen as

$$
L_{1}=\left[\begin{array}{cc}
0.0489 & -0.0210 \\
-0.203 & -0.0191
\end{array}\right], \quad L_{2}=\left[\begin{array}{cc}
-0.0240 & 0.0008 \\
0.1333 & 0.0631
\end{array}\right], \quad r(t)=3
$$

By solving LMI (15), the observer gain matrix is computed as

$$
K=\left[\begin{array}{cc}
-0.5627 & 0.3505 \\
0.1952 & -0.1210
\end{array}\right]
$$

and the other parameters are obtained: $\varepsilon=127.07, \varsigma=0.3$. To illustrate the effectiveness of the proposed adaptive observer, consider loss-of-effectiveness fault model, i.e., the system operates in the normal case before $30 \mathrm{~s}$, and both $u_{1}(t)$ and $u_{2}(t)$ lose $50 \%$ of the effectiveness at $30 \mathrm{~s}$, which means that parameters $\theta_{1}$ and $\theta_{2}$ become -0.5 .

The trajectories of states $x_{1}(t)$ and $x_{2}(t)$ together with their estimations of system (2) are shown in lower parts of Figs. 1 and 2, respectively. The fault parameters $\theta_{1}$ and $\theta_{2}$ together with their estimation are shown in lower parts of Figs. 3 and 4, respectively.
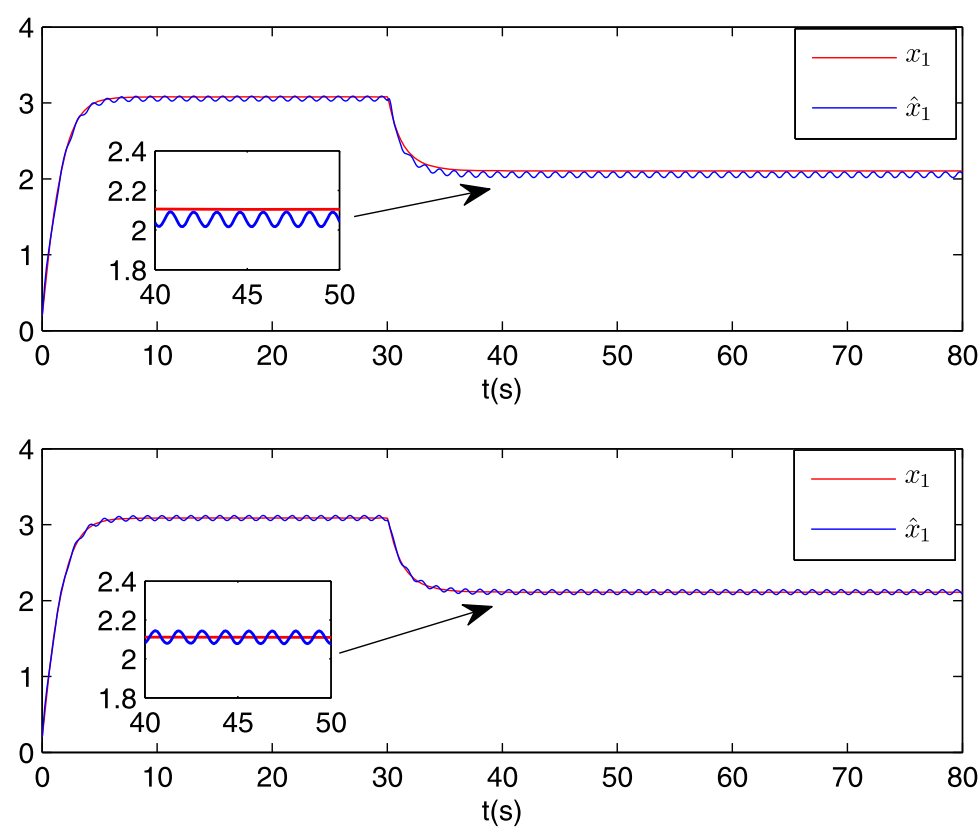

Figure 1 State $x_{1}(t)$ and its estimation. The blue line denotes state $x_{1}(t)$ while the red line denotes its estimation. The lower part is the result obtained using method proposed in this paper, while the upper part is the result obtained using method proposed in [11] 

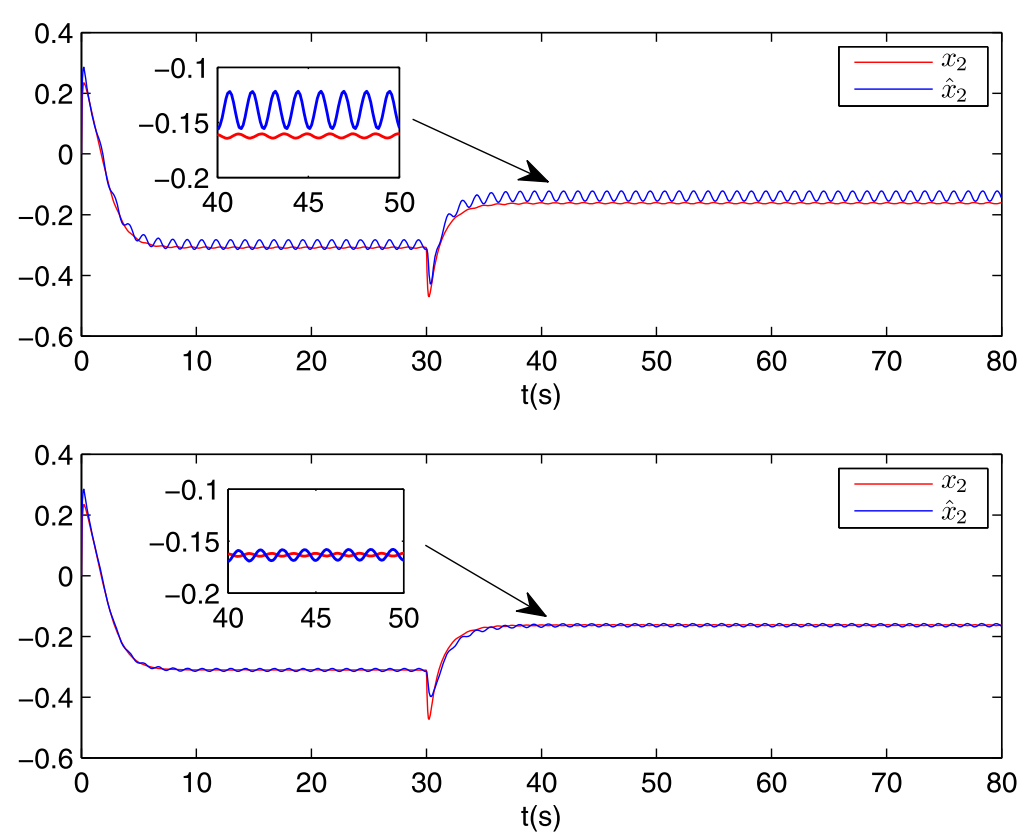

Figure 2 State $x_{2}(t)$ and its estimation. The blue line denotes state $x_{2}(t)$ while the red line denotes its estimation. The lower part is the result obtained using method proposed in this paper, while the upper part is the result obtained using method proposed in [11]
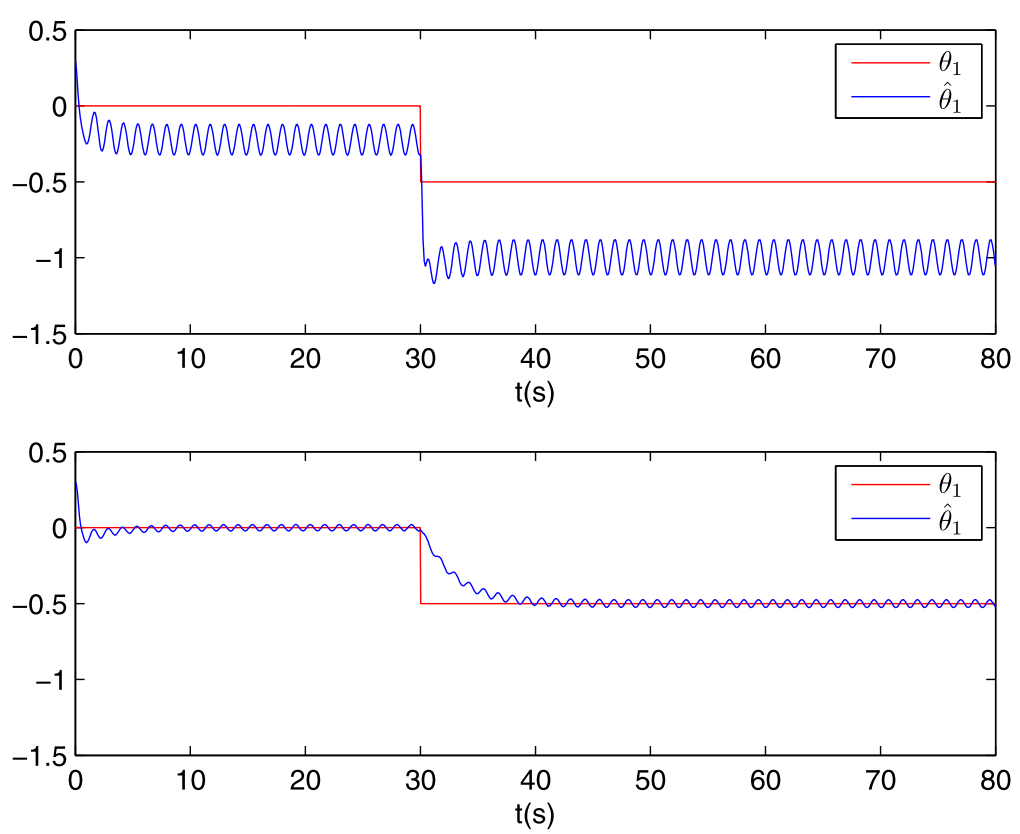

Figure 3 Actuator fault parameter $\theta_{1}(t)$ and its estimation. The red line denotes state $\theta_{1}(t)$ while the blue line denotes its estimation. The lower part is the result obtained using method proposed in this paper, while the upper part is the result obtained using method proposed in [11]

To make it more convincing, the proposed approach is compared with the adaptive fuzzy observer proposed in [11] where premise variables are assumed to be measurable. For the same system and fault models, the simulation results are shown in the upper parts of 

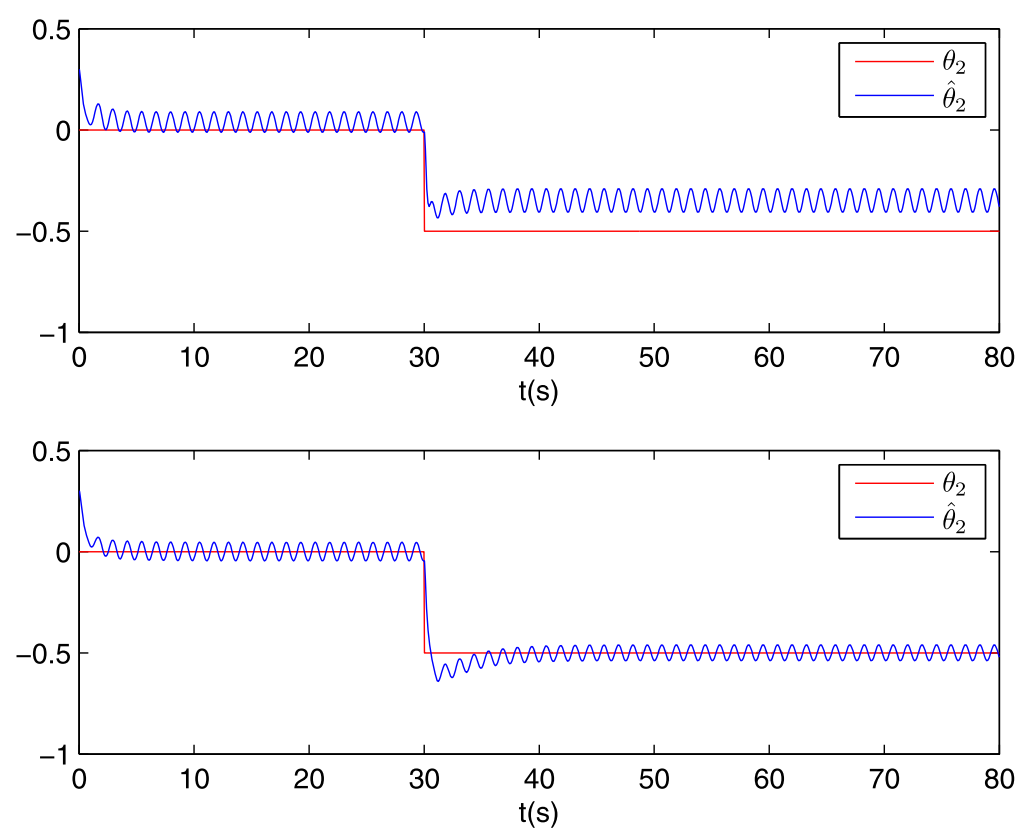

Figure 4 Actuator fault parameter $\theta_{2}(t)$ and its estimation. The red line denotes state $\theta_{2}(t)$ while the blue line denotes its estimation. The lower part is the result obtained using method proposed in this paper, while the upper part is the result obtained using method proposed in [11]

Figs. 1, 2, 3, 4. From Figs. 1, 2, 3, 4, it can be seen that the adaptive fuzzy observer with unmeasurable premise variables proposed in this paper gets better estimation results.

\section{Conclusions}

This paper focuses on the fault estimation problem for a class of T-S fuzzy system with actuator faults and sensor disturbances. Premise variables are assumed to be unmeasurable, through designing an adaptive observer, states and actuator fault parameters are estimated simultaneously. It has been proved that the state and fault estimation errors are both uniformly ultimately bounded. Finally, a numerical example is given to illustrate the effectiveness of the proposed methods.

Acknowledgements

This work was supported in part by the Funds of National Science of China (Grant No. 61673098).

Competing interests

The authors declare that they have no competing interests.

Authors' contributions

All authors contributed equally in writing this paper. All authors read and approved the final manuscript.

\section{Publisher's Note}

Springer Nature remains neutral with regard to jurisdictional claims in published maps and institutional affiliations.

Received: 12 September 2017 Accepted: 20 March 2018 Published online: 27 March 2018

References

1. Isermann, R.: Supervision, fault-detection and fault-diagnosis methods-An introduction. Control Eng. Pract. 5, 639-652 (1997)

2. Yin, S., Ding, S.X., Zhou, D.H.: Diagnosis and prognosis for complicated industrial systems-Part II. IEEE Trans. Ind. Electron. 63, 3201-3204 (2016) 
3. Li, H.Y., Chen, Z.R., Wu, L.G., Lam, H.K., Du, H.P.: Event-triggered fault detection of nonlinear networked systems. IEEE Trans. Cybern. 47, 1041-1052 (2017)

4. Wu, L., Yao, X., Zheng, W.X.: Generalized $\mathrm{H}_{2}$ fault detection for Markovian jumping two-dimensional systems. Automatica 48, 1741-1750 (2012)

5. Zhai, D., Lu, A.Y., Li, J.H., Zhang, Q.L.: Simultaneous fault detection and control for switched linear systems with mode-dependent average dwell-time. Appl. Math. Comput. 273, 767-792 (2016)

6. Li, X.J., Yang, G.H.: Fault detection for T-S fuzzy systems with unknown membership functions. IEEE Trans. Fuzzy Syst. 22, 139-152 (2014)

7. Li, L.L., Ding, S.X., Qiu, J.B., Yang, Y., Xu, D.M.: Fuzzy observer-based fault detection design approach for nonlinear processes. IEEE Trans. Syst. Man Cybern. Syst. 47, 1941-1952 (2017)

8. Zhang, X.D., Polycarpou, M.M., Parisini, T.: Fault diagnosis of a class of nonlinear uncertain systems with Lipschitz nonlinearities using adaptive estimation. Automatica 46, 290-299 (2010)

9. Yang, G.H., Wang, H.: Fault detection for a class of uncertain state-feedback control systems. IEEE Trans. Control Syst. Technol. 18, 201-212 (2010)

10. Yan, X.G., Edwards, C.: Nonlinear robust fault reconstruction and estimation using a sliding mode observer. Automatica 43, 1605-1614 (2007)

11. Jiang, B., Staroswiecki, M., Cocquempot, V.: Fault diagnosis based on adaptive observer for a class of non-linear systems with unknown parameters. Int. J. Control 77, 415-426 (2004)

12. Li, X.J., Yang, G.H.: Fault diagnosis for non-linear single output systems based on adaptive high-gain observer. IET Control Theory Appl. 7, 1969-1977 (2013)

13. Chadli, M., Karimi, H.R.: Robust observer design for unknown inputs Takagi-Sugeno models. IEEE Trans. Fuzzy Syst. 21, 158-164 (2013)

14. Zhang, K., Jiang, B., Shi, P.: Fault estimation observer design for discrete-time Takagi-Sugeno fuzzy systems based on piecewise Lyapunov functions. IEEE Trans. Fuzzy Syst. 20, 192-200 (2012)

15. Youssef, T., Karimi, H.R., Chadli, M.: Faults diagnosis based on proportional integral observer for TS fuzzy model with unmeasurable premise variable. In: 2014 IEEE International Conference on Fuzzy Systems (FUZZ-IEEE), pp. 2119-2124 (2014)

16. Lendek, Z., Lauber, J., Guerra, T.M., Babuska, R., Schutter, B.: Adaptive observers for TS fuzzy systems with unknown polynomial inputs. Fuzzy Sets Syst. 161, 2043-2065 (2010)

17. Jiang, B., Zhang, K., Shi, P.: Integrated fault estimation and accommodation design for discrete-time Takagi-Sugeno fuzzy systems with actuator faults. IEEE Trans. Fuzzy Syst. 19, 291-304 (2011)

18. Tanaka, K., Wang, H.O.: Fuzzy Control Systems Design and Analysis: A LMI Approach. Wiley, New York (2001)

19. Zhao, Y., Lam, J., Gao, H.: Fault detection for fuzzy systems with intermittent measurements. IEEE Trans. Fuzzy Syst. 17 398-410 (2009)

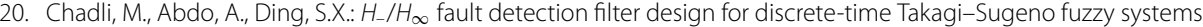
Automatica 49, 1996-2005 (2013)

21. Aouaouda, S., Chadli, M., Cocquempot, V., Khadir, M.T.: Multi-objective $H_{-} / H_{\infty}$ fault detection observer design for Takagi-Sugeno fuzzy systems with unmeasurable premise variables: descriptor approach. Int. J. Adapt. Control Signal Process. 27, 1031-1047 (2013)

22. Tao, G., Joshi, S.M., Ma, X.: Adaptive state feedback and tracking control of systems with actuator failures. IEEE Trans. Autom. Control 46, 78-95 (2001)

23. Li, X.J., Yang, G.H.: Adaptive fault-tolerant synchronization control of a class of complex dynamical networks with general input distribution matrices and actuator faults. IEEE Trans. Neural Netw. Learn. Syst. 28, 559-569 (2017)

24. Zhang, Q.H.: Adaptive observer for multiple-input-multiple-output (MIMO) linear time-varying systems. IEEE Trans. Autom. Control 47, 525-529 (2002)

25. Xu, A.P., Zhang, Q.H.: Nonlinear system fault diagnosis based on adaptive estimation. Automatica 40, 1181-1193 (2004)

26. Xu, A.P., Zhang, Q.H.: Residual generation for fault diagnosis in linear time-varying systems. IEEE Trans. Autom. Control 49, 767-772 (2004)

27. Youssef, T., Chadli, M., Karimi, H.R., Wang, R.: Actuator and sensor faults estimation based on proportional integral observer for TS fuzzy model. J. Franklin Inst. 354, 2524-2542 (2017)

\section{Submit your manuscript to a SpringerOpen ${ }^{\circ}$ journal and benefit from:}

- Convenient online submission

- Rigorous peer review

- Open access: articles freely available online

- High visibility within the field

- Retaining the copyright to your article

Submit your next manuscript at $\gg$ springeropen.com 